\title{
Research on Clinical Characteristics and Prognostic Analysis of Heparin-Induced Thrombocytopenia After Surgery for Acute Type a Aortic Dissection
}

\section{Chu-zhi Zhou}

FuWai Hospital,Chinese Academy of Medical Sciences,Shenzhen

\section{Yan-zhen Li}

Tianjin Medical University

Dong-jie Feng

Fuwai Hospital,Chinese Academy of Medical Siences,Shenzhen

\section{Yuan Fang}

Fuwai Hospital,Chinese Academy of Medical Sciences,Shenzhen

\section{Feng-yan Zha}

Fuwai Hospital,Chinese Academy of Medical Sciences,Shenzhen

\section{Erhui Wang}

Fuwai Hospital,Chinese Academy of Medical Sciences,Shenzhen

\section{Min-xin Wei ( $239196788 @ q q . c o m$ )}

Graduate School of Tianjin Medical University

Jun-min Wen

Fuwai Hospital,Chinese Academy of Medical Sciences,Shenzhen

\section{Research article}

Keywords: Blood platelet count, heparin-induced thrombocytopenia, thrombosis, acute aortic dissection, heparin

Posted Date: December 1st, 2020

DOl: https://doi.org/10.21203/rs.3.rs-114646/v1

License: (c) (i) This work is licensed under a Creative Commons Attribution 4.0 International License. Read Full License

Version of Record: A version of this preprint was published at Journal of Cardiothoracic Surgery on April 20th, 2021. See the published version at https://doi.org/10.1186/s13019-021-01482-2. 


\title{
Research on clinical characteristics and prognostic analysis of heparin-induced thrombocytopenia after surgery for acute type A aortic dissection
}

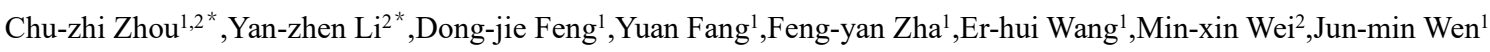 \\ ${ }^{1}$ Department of Critical Care Medicine ICU,Fu Wai Hospital, Chinese Academy of Medical Sciences, Shenzhen, \\ Shenzhen,518057, Guangdong,China. \\ ${ }^{2}$ Graduate School of Tianjin Medical University, Tianjin,300070, China \\ ${ }^{*}$ Both authors contributed equally to this work. \\ Corresponding author:Min-xin Wei, Email: 239196788@qq.com.Jun-min Wen,1029013908@qq.com
}

\begin{abstract}
Purpose: The present study aimed to explore the clinical characteristics of heparin-induced thrombocytopenia (HIT) after surgery for acute type A aortic dissection and perform a relevant prognostic analysis.
\end{abstract}

Methods: After continuous observation and analysis of 204 patients who underwent acute type A aortic dissection, we found that blood platelets decreased significantly after surgery and that these patients can be suspected to suffer HIT based on relevant 4 Ts scores. For these suspected HIT patients, a latex particle-enhanced immunoturbidimetric assay was conducted to detect heparin-induced antibodies. Perioperative clinical data of patients in HIT and non-HIT groups were recorded as were blood platelet counts, HIT antibody test results, 4Ts scores, thromboembolic complications, clinical prognosis and outcomes.

Results: In the present study, 38 suspected HIT patients, 16 HIT patients and 188 non-HIT patients were selected in the clinical setting. Among them, HIT patients were found to have prolonged cardiopulmonary bypass time(223min on average vs. 164min) and delayed aortic cross-clamp time(128min on average vs. $107 \mathrm{~min})$, and these differences between HIT patients and non-HIT patients were significant $(\mathrm{P}<0.05)$. Additionally, the HIT group required longer operation time and higher dose of heparin, but showing no statistical differences $(\mathrm{P}>0.05)$. The transfusions of blood platelets in the HIT 
group and non-HIT group were $18.7 \pm 5.0 \mathrm{u}$ and $15.6 \pm 7.34 \mathrm{u}$, respectively.In the HIT group, the mechanic ventilation time and the length of ICU stay were longer comparing the non-HIT group $(\mathrm{P}<0.05)$, though no significant differences in total length of stay or In-hospital mortality were observed $(\mathrm{P}>0.05)$. The incidence of continuous renal replacement therapy in HIT group was higher than the non-HIT group $(\mathrm{P}<0.05)$. Additionally,there were no significant differences in 24 -h postoperative drainage or reoperation for bleeding in both group $(\mathrm{P}>0.05)$. However, the HIT antibody titer in the HIT group was significantly higher than that in the Suspected HIT group $(2.7 \pm 1.8 \mathrm{U} / \mathrm{mL}$ vs. $0.3 \pm 0.2 \mathrm{U} / \mathrm{mL})(\mathrm{P}<0.05)$.Among patients diagnosed with HIT, the incidence of thromboembolism reached 31.5\%.For example, two HIT patients newly developed thromboembolism in both lower extremities,and three patients experienced cerebral infarction.

Conclusions: After surgery for acute type A aortic dissection, HIT patients developed postoperative complications, the duration of ventilatory support and length of ICU stay were extended, and the incidence of thromboembolism increased. HIT antibody detection and risk classification should be implemented for high-risk patients showing early clinical characteristics.

Keywords: Blood platelet count; heparin-induced thrombocytopenia; thrombosis; acute aortic dissection; heparin

\section{INTRODUCTION}

Heparin-induced thrombocytopenia (HIT) is an iatrogenic disease that potentially threatens the lives of people exposed to heparin or low-molecular-weight heparin. Because of the use of heparin during cardiopulmonary bypass, patients undergoing cardiac surgeries are at risk of $\mathrm{HIT}^{[1]}$. For example, risk of thrombosis mediated by heparin platelet factor 4 (PF-4) complex antibody in the circulation was found to be 30 times higher than that of the general population ${ }^{[2]}$. A reduction in blood platelet count (PLTc) after surgery for acute type A aortic dissection commonly occurs in the clinical setting. However, HIT induced by heparin during cardiopulmonary bypass is rarely reported. Furthermore, no specificity in clinical manifestations of HIT has been found, which mainly include PLTc decrease at several days or months after heparin therapy along with the presence or absence of a newly developed thromboembolism. In this case, a contradictory state between a decrease in PLTc and thrombosis occurs. This poses a great challenge to clinical treatment ${ }^{[3]}$. In this study, patients undergoing acute type A 
aortic dissection were observed for signs of HIT, and clinical outcomes after surgery were assessed. Hopefully, appropriate support can be provided for clinical prevention and prognosis.

\section{METHODS}

\section{Study design}

Patients with suspected HIT undergoing acute type A aortic dissection were continuously observed from August 2018 to December 2019 in our hospital, Chinese Academy of Medical Sciences. ethics committee approval (Institutional Review Board File 201810), signed the clinical research informed consent, The following inclusion criteria were applied:(1) severe chest pain and acute type A aortic dissection confirmed by computed tomography;(2)a PLTc decrease above 30\% (compared with its baseline value before surgery) after the administration of heparin during the extracorporeal circulation; (3) complete clinical data. When a patient is suspected to have HIT in the clinic, 4Ts scores should be obtained $^{[4]}$; if the score is $\geq 4$ points, an antiplatelet factor 4-heparin antibody (HIT antibody) should be tested.

\section{Laboratory assays}

HIT antibody detection: HIT-Ab (PF4-H) in human citrate plasma was examined by a fully automatic latex-enhanced immunoturbidimetric assay using an ACL TOP 300 (Werfen, USA). Anti-heparin antibodies in HIT patients were detected using latex particle-enhanced immunoturbidimetric assays. Regarding samples collected from patients undergoing heparin therapy, the results may be considered positive if the HIT antibody detection kit shows that the corresponding value is no less than a threshold of $1.0 \mathrm{U} / \mathrm{mL}$. This signifies the presence of HIT antibodies ${ }^{[5]}$.

Collection of clinical data: (1) The baseline information of all hospitalized patients with acute type A aortic dissection, including sex, age, body mass index, medical history (e.g.hypertension, diabetes, smoking or severe COPD), continuous renal replacement therapy (CRRT) or not, preoperative ejection fraction and PLTc, were obtained. (2) Data on the time of extracorporeal circulation, time of aortic occlusion, time of circulation arrest, volume of blood platelets transfused during the surgery and heparin dosage were collected. (3) Complications during hospitalization and prognosis of all patients were also recorded. 


\section{Statistical analysis}

Statistical analysis was conducted using SPSS 19.0 software. Continuous variables with normal distribution are expressed as $\mathrm{x} \pm \mathrm{s}$; variables with nonnormal distribution are expressed as medians and the $25 \%$ or $75 \%$ fractile.Categorical variables are expressed as percentages. To compare baseline information, an unpaired t-test and a chi-square test were conducted. Statistically significant differences were considered at a $\mathrm{P}$ value $<0.05$.

\section{RESULTS}

A total of 204 patients with acute type A aortic dissection were consecutively included and observed. They were grouped according to 4 Ts scores, and the 38 patients with a score of $\geq 4$ points were initially classified as the suspected HIT group. Among these suspected HIT patients, some of them were eventually diagnosed with HIT through HIT antibody detection. Specifically, 16 and 188 patients were included in the HIT and non-HIT groups, respectively. The results of the comparison between the two groups are presented in Tables 1, 2 and 3. Through comparison of preoperative data, no significant differences were observed in age, sex or body mass index between the HIT and non-HIT groups $(\mathrm{P}>0.05)$. Moreover, the HIT group was not significantly different from the non-HIT group in terms of the incidence of hypertension, diabetes, history of smoking, severe COPD or ejection fraction $(\mathrm{P}>0.05)$. Moreover, patients in the HIT group had prolonged cardiopulmonary bypass time $(223 \mathrm{~min}$ on average vs.164 $\mathrm{min}, \mathrm{P}<0.01)$ and a long cross-clamping period (128 min on average vs.107 min, $\mathrm{P}<0.05)$, with significance $(\mathrm{P}<0.05)$. Although prolonged cardiopulmonary bypass time and a comparatively higher dose of heparin were observed in the HIT group, no significant difference from the dose of heparin in the non-HIT group was observed $(\mathrm{P}>0.05)$. Furthermore, no significant differences were found in the volumes of blood platelets transfused between the two groups $(18.7 \pm 5.0 \mathrm{u}$ vs. $15.6 \pm 7.34 \mathrm{u})$. The mechanical ventilation time and length of ICU stay were longer in the HIT group, which was significantly different from those in the non-HIT group $(\mathrm{P}<0.05)$. However, no significant differences in the total length of stay or the all-cause mortality were found between the two groups. Stroke incidence and CRRT occurrence rates in the HIT group were higher than those in the non-HIT group $(\mathrm{P}<0.05)$. Twenty-four hours after the surgery, no significant differences were found in the drainage fluid amounts or second thoracotomy. Moreover, the HIT antibody titer in 
the HIT group $(2.7 \pm 1.8 \mathrm{U} / \mathrm{mL})$ was much greater than that in the suspected HIT group $(0.3 \pm 0.2 \mathrm{U} / \mathrm{mL})$ (Table 4, $\mathrm{P}<0.05)$. In addition, the HIT and suspected HIT groups presented no significant differences in terms of 4Ts scores and minimum PLT and PLT decrease. Regarding the 16 patients in the HIT group, heparin or low-molecular-weight heparin treatment was immediately discontinued when HIT was suspected during evaluation in the clinic. Moreover, the agents used for monitoring arterial tube washing in 38 patients with suspected cases were replaced with argatroban.

Table 1. Preoperative data comparison between HIT and non-HIT groups

\begin{tabular}{lllll}
\hline Parameters & $\begin{array}{l}\text { All patients } \\
(\mathrm{n}=204)\end{array}$ & $\begin{array}{l}\text { HIT group } \\
(\mathrm{n}=16)\end{array}$ & $\begin{array}{l}\text { Non-HIT group } \\
(\mathrm{n}=188)\end{array}$ & P value \\
\hline Age(years) & $51.0 \pm 13.1$ & $52.0 \pm 13.3$ & $51.5 \pm 13.0$ & 0.879 \\
Sex (M/F) & $168 / 36$ & $10 / 6$ & $158 / 30$ & 0.737 \\
BMI(kg/m²) & $24.8 \pm 3.8$ & $25.5 \pm 2.5$ & $24.8 \pm 3.9$ & 0.112 \\
Hypertension,n (\%) & $141(69.1)$ & $11(68.8)$ & $130(69.1)$ & 0.816 \\
DM,n (\%) & $22(10.7)$ & $2(12.5)$ & $20(10.6)$ & 0.560 \\
History of smoking & $82(40.2)$ & $5(31.3)$ & $77(40.9)$ & 0.343 \\
$\begin{array}{l}\text { Renal replacement therapy,n } \\
(\%)\end{array}$ & $0(0)$ & $0(0)$ & $0(0)$ & 1.000 \\
Preoperative platelet & $195.8 \pm 79.8$ & $212.9 \pm 67.3$ & $192.8 \pm 82.5$ & 0.211 \\
count $(\times 10 \%$ L) & & & & \\
COPD,n (\%) & $3(1.5)$ & $0(0)$ & $5(1.6)$ & 0.584 \\
LVEF (\%) & $57.8 \pm 7.8$ & $60.2 \pm 3.2$ & $57.7 \pm 7.7$ & 0.201 \\
\hline
\end{tabular}

BMI, body mass index; DM, diabetes mellitus; COPD, chronic obstructive pulmonary disease; LVEF, left ventricular ejection fraction;

Table 2. Intraoperative data comparison between the HIT group and the non-HIT group

\begin{tabular}{lllll}
\hline Parameters & All patients $(\mathrm{n}=204)$ & $\begin{array}{l}\text { HIT } \\
\operatorname{group}(\mathrm{n}=16)\end{array}$ & $\begin{array}{l}\text { Non-HIT } \\
\text { group(n=188) }\end{array}$ & P value \\
\hline Renal replacement therapy,n & $16(7.8)$ & $7(43.8)$ & $9(4.8)$ & 0.001
\end{tabular}


$(\%)$

\begin{tabular}{lllll} 
In-hospital mortality,n (\%) & $10(4.9)$ & $2(12.5)$ & $8(4.3)$ & 0.622 \\
$\begin{array}{l}\text { Postoperative stroke,n (\%) } \\
\begin{array}{l}\text { Mechanic ventilation time } \\
\text { (hours) }\end{array}\end{array}$ & $10(4.9)$ & $4(25)$ & $6(3.2)$ & 0.001 \\
$\begin{array}{l}\text { Length of ICU stay(hours) } \\
\begin{array}{l}\text { 24-h postoperative } \\
\text { drainage(mL) }\end{array}\end{array}$ & $142.7 \pm 123.2$ & $226.5 \pm 136.9$ & $126.6 \pm 110.9$ & 0.012 \\
$\begin{array}{l}\text { Length of in-hospital(days) } \\
\text { Reoperation for bleeding,n(\%) }\end{array}$ & $14(6.8)$ & $536.9 \pm 222.1$ & $537.1 \pm 384.7$ & 0.997 \\
\hline
\end{tabular}

Table 3. Postoperative data comparison between HIT and non-HIT groups

\begin{tabular}{lllll}
\hline Parameters & $\begin{array}{l}\text { All patients } \\
(\mathrm{n}=204)\end{array}$ & $\begin{array}{l}\text { HIT group } \\
(\mathrm{n}=16)\end{array}$ & $\begin{array}{l}\text { Non-HIT group } \\
(\mathrm{n}=188)\end{array}$ & P value \\
\hline CPB time(min) & $175.8 \pm 63.6$ & $223.1 \pm 11.4$ & $164.9 \pm 59.8$ & 0.006 \\
Cross-clamp time(min) & $111.8 \pm 42.4$ & $128.9 \pm 30.1$ & $107.1 \pm 45.5$ & 0.042 \\
DHCA time(min) & $17.9 \pm 7.0$ & $20.6 \pm 7.1$ & $16.6 \pm 7.1$ & 0.085 \\
Intraoperative platelet(unit) & $16.0 \pm 6.6$ & $18.7 \pm 5.0$ & $15.6 \pm 7.3$ & 0.131 \\
Heparin dose(mg) & $336.1 \pm 80.2$ & $407.1 \pm 192.9$ & $325.9 \pm 77.2$ & 0.081 \\
\hline
\end{tabular}

CPB, cardiopulmonary bypass; DHCA, deep hypothermia circulatory arrest;

Table 4. Comparison of HIT and suspected HIT groups

\begin{tabular}{lcccc}
\hline Parameters & $\begin{array}{c}\text { All patients } \\
(n=38)\end{array}$ & $\begin{array}{c}\text { HIT group } \\
(n=16)\end{array}$ & $\begin{array}{l}\text { Suspected } \\
\text { HIT group } \\
(n=22)\end{array}$ & $\begin{array}{l}\text { P } \\
\text { value }\end{array}$ \\
\hline 4Ts score & $5.4 \pm 1.2$ & $5.7 \pm 1.2$ & $5.3 \pm 1.1$ & 0.527 \\
HIT antibody titer $(\mathrm{U} / \mathrm{mL})$ & $1.4 \pm 1.6$ & $2.7 \pm 1.8$ & $0.3 \pm 0.2$ & 0.001 \\
PLT drop $(\%, 95 \% \mathrm{CI})$ & $70.1(67.9 \sim 79.0)$ & $73.6(67.2 \sim 79.4)$ & $67.9(62.9 \sim 71.7)$ & 0.236 \\
Minimum PLT $\left(10^{9} / \mathrm{L}\right)$ & $44.4 \pm 16.6$ & $40.9 \pm 17.2$ & $47.6 \pm 20.1$ & 0.076 \\
\hline
\end{tabular}




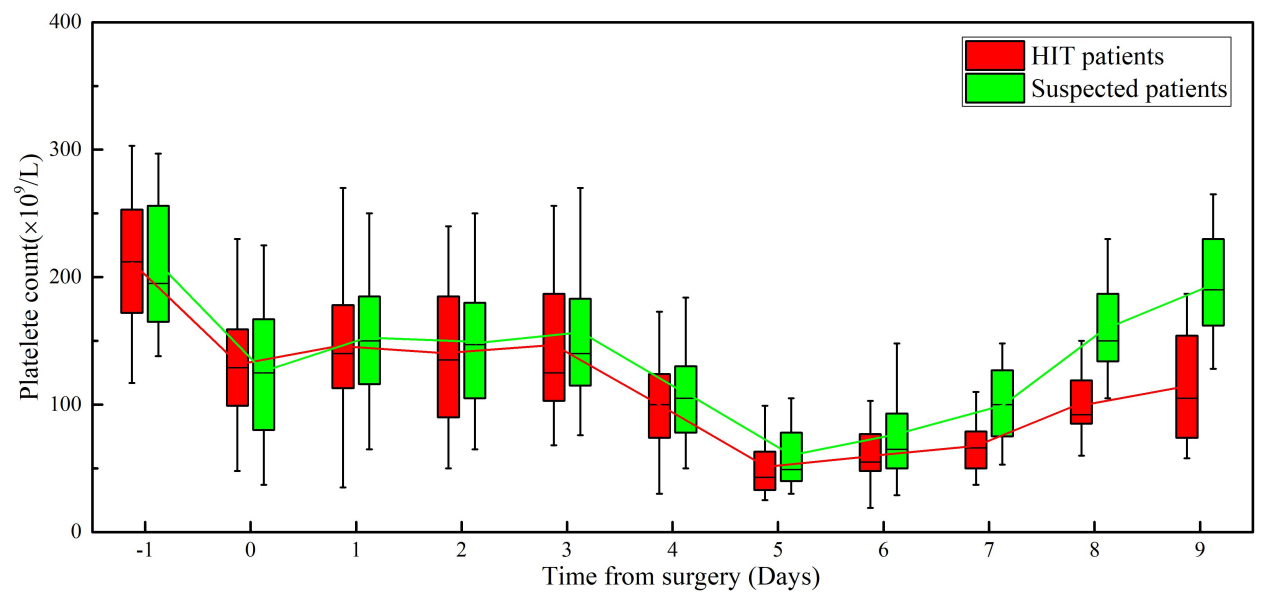

Figure 1:Comparison of platelet counts between HIT patients and Suspected HIT patients.

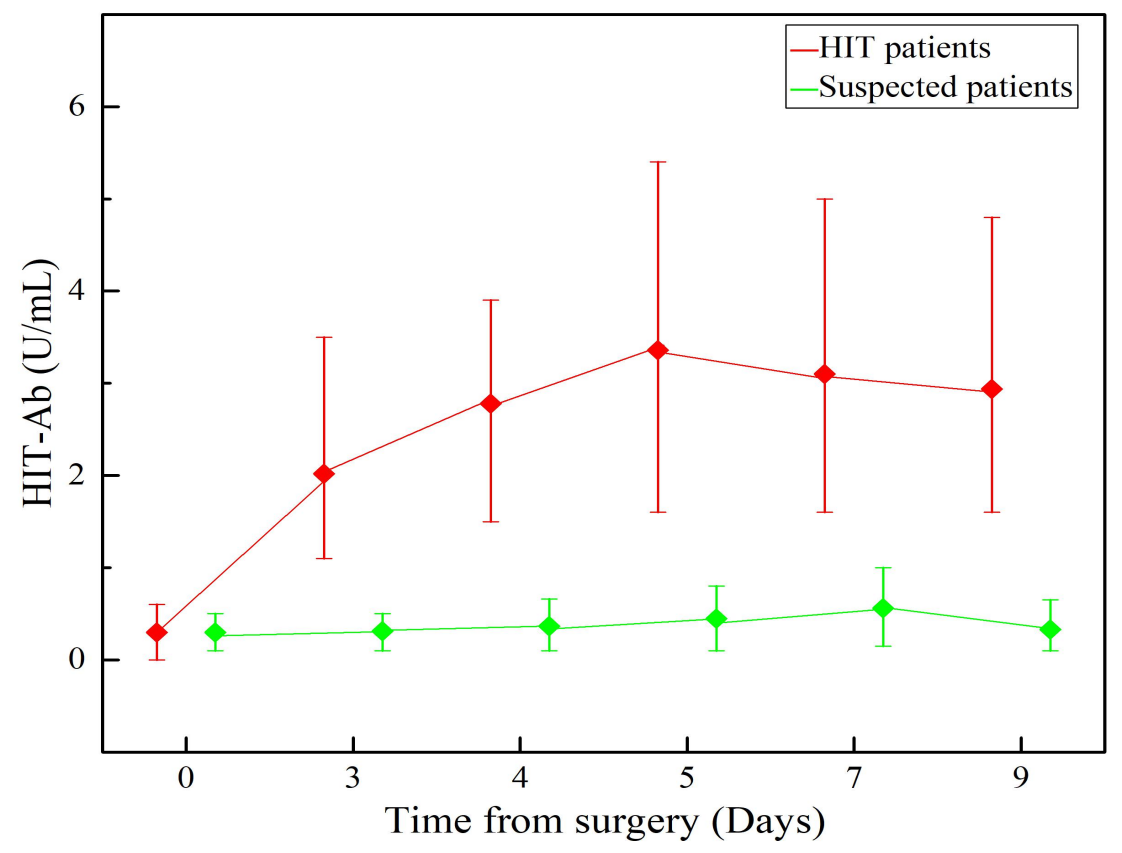

Figure 2:Comparison of HIT-Ab between HIT patients and Suspected HIT patients.

\section{DISCUSSION}

The abovementioned data were collected from patients who underwent surgery in the aorta. In our study,the incidence of HIT was $7.8 \%(16 / 204)$ for specific patients who underwent surgery under deep hypothermic circulatory arrest(DHCA). Regarding thoracotomy, extracorporeal circulation is associated with the anticoagulation process of heparin. For this reason, patients with acute type A aortic dissection 
undergo a longer procedure and a short DHCA process during surgery. All patients in this series were exposed to a large dose of heparin, and postoperative PLTc reduction for unknown causes increases the risk for HIT development. HIT onset is not easy to perceive due to a lack of specificity in clinical manifestations, though a PLTc reduction 3 days after heparin administration is the most common. Occasionally, such a manifestation may be observed within 24 hours or several months after the application of heparin ${ }^{[6]}$.

The findings of the present study strengthen the awareness that HIT is a important complication worth causing our great attention after surgery for acute type A aortic dissection. Although the differences in preoperative data between the HIT group and the non-HIT group showed no statistical significance. Because of apparently longer extracorporeal circulation time and aortic cross-clamp time in the HIT group, the dose of heparin needs to be increased. In our study, the doses of heparin in both groups were not significantly different from each other $(\mathrm{P}>0.05)$. The extension of the time of extracorporeal circulation may enable the body to be exposed to heparin for a longer time. Hence, achieving an appropriate ratio between heparin and PF-4 and produce pathogenic HIT antibodies becomes much more likely ${ }^{[7]}$. In this context, the time of extracorporeal circulation and aortic cross-clamp should be reduced to the greatest extent during surgery for acute type A aortic dissection. Thus, both the exposure dose of heparin and the time of exposure can be decreased. PLTc reduction induced by HIT after surgery for acute type A aortic dissection makes it more possible to administer renal replacement therapy after acute kidney injury. The pathogenesis might involve the influence of microvascular thrombosis development because microvascular thrombosis has the potential to reduce the volume of blood flow in the kidney, further lowering the glomerular filtration rate and finally leading to acute kidney injury[8]. Although HIT increases the rate of stroke incidence, both thromboembolism and microvascular blood flow disorder are the primary reasons why the risk of stroke increases in this group. Nonetheless, further investigations are warranted to identify the effects of PLTc reduction on AKI and stroke after cardiopulmonary bypass. Considering that a longer extracorporeal circulation time is also a critical cause of an increase in postoperative complications, corresponding causal relationships or interactions should be further explored and clarified as well. Both the duration of mechanic ventilation and length of ICU stay were apparently longer in the HIT group.Although the differences in the length of in-hospital were eventually found to be insignificant between the HIT and 
non-HIT groups and no statistical differences in in-hospital mortality rates were observed, the relevant treatment costs and risks increased. In contrast, HIT reduces the PLTc, but the $24 \mathrm{~h}$ postoperative volume of drainage fluid has not significant increase, and the probability of reoperation for bleeding does not increase at the same time, which may be related to the characteristics of HIT, that there are contradictions between PLTc reduction and thrombosis),conversely, the risk of hemorrhage does not increase.

HIT occurs along with arterial and venous thrombotic events; newly developed thromboembolism is the most serious clinical outcome of HIT, primarily including thrombosis in the lower limbs, pulmonary embolism, myocardial infarction, cerebral infarction, local necrosis in the heparin-injected site, gangrene, necrosis and hemorrhage in the adrenal gland ${ }^{[9]}$. Among the patients included in the present study, two patients had new-onset thromboembolism in both lower limbs, and three patients had postoperative cerebral infarction. The thromboembolism incidence rate reached $31.5 \%$, greatly similar to the ratios $(30 \%-50 \%)$ reported in other countries ${ }^{[10]}$. If the PLTc decreases by over $50 \%$ or if thrombosis occurs, then suspected HIT is confirmed ${ }^{[11]}$. The 4 Ts scoring system combines the following four characteristics: (1) amplitude of PLTc reduction, (2) duration of PLTc reduction after exposure to heparin, (3) presence of thrombosis,(4) absence of PLTc reduction due to other causes ${ }^{[12]}$. Such a system has been widely applied in the clinical setting. A score below four points indicates a high negative predictive value $(97 \%-100 \%)$, whereas that above six points suggests that the corresponding positive predictive value is low $(40 \%-82 \%)^{[13]}$. Clearly, the 4 Ts scoring system has high sensitivity but low specificity, though it can be used as a preliminary screening method for exclusion of HIT in the clinical setting.

PLTc reduction is very common after surgery for acute type A aortic dissection, and true and false lumens are formed in the aorta after the onset of aortic dissection. Consequently, the blood flows through the intimal tear into the false lumen and thus comes into contact with the subendothelial tissues. In this way, coagulation and fibrinolysis systems are activated, leading to platelet activation and aggregation as well as the formation of blood $\operatorname{clots}^{[14]}$. In addition to extensive thrombosis in the false lumen, microthrombogenesis occurs in the whole body, causing the consumption of massive blood platelets and other blood coagulation factors. Therefore, both PLTc reduction and extensive thrombosis in the false lumen are closely related to platelet consumption ${ }^{[15]}$.The existing literature notes that 
expression of platelet-activating factor-4 is upregulated in the early stage of acute type A aortic dissection ${ }^{[16]}$. However, the correlation between platelet-activating factor-4 expression upregulation and HIT remains unclear. Regardless, there is no doubt that such upregulation increases the likelihood of binding after heparin exposure, which may be a reason for the occurrence of HIT. During extracorporeal circulation, the interaction of blood and the tube, together with the shear force of the loop, can lead to platelet activation. As a consequence, the blood constituents in peripheral circulation are consumed ${ }^{[17]}$, though the platelet aggregation function may change after extracorporeal circulation. Accordingly, variations in surface marker expression, morphology and volume in blood platelets can be found ${ }^{[18]}$. Furthermore, PLTc reduction is associated with the administration of antiplatelet drugs, and 4Ts scores are therefore elevated. This may cause an increase in false-positive rates obtained through HIT diagnosis. Through 4Ts scoring, 38 patients in this study were suspected to have HIT, and these patients were divided into confirmed and suspected HIT groups based on laboratory testing of HIT antibodies. As shown in Table 4, the 4Ts scores of the confirmed HIT group showed no differences from those of the suspected HIT group. In terms of clinical manifestations, no significant differences in either absolute values of minimum PLT or PLT reduction were observed. Considering that the 4Ts scoring system has high sensitivity but low specificity ${ }^{[19]}$, it should only be used as a preliminary screening method for preliminary exclusion of HIT in the clinical setting. Because of low specificity, HIT antibody detection needs to be further implemented to avoid overdiagnosis and incorrect treatment.

As seen from the trend illustrated in Figure 1 and Figure 2, the HIT antibody concentration in the HIT group reached its peak on the fifth day after surgery,when the platelet count reduced at its lowest.Furthermore, the concentration of HIT antibodies in the confirmed HIT group $(2.7 \pm 1.8 \mathrm{U} / \mathrm{mL})$ was significantly higher than that of the Suspected HIT group $(0.3 \pm 0.2 \mathrm{U} / \mathrm{mL})$, providing a valid basis for early diagnosis of HIT in the clinical setting. Currently, the corresponding diagnostic measures principally include specific anti-HIT-IgG antibody and anti-HIT antibody complexes (e.g., IgG, IgA and $\operatorname{IgM})^{[20]}$. Enzyme-linked immunosorbent assays, particle immune approaches and colloidal gold immunochromatography are commonly used manual testing methods. In our study, immunonephelometry was used to achieve rapid detection with a fully automatic coagulometer (15 $\min )^{[21]}$. The negative predictive value obtained through antibody detection is nearly $99 \%{ }^{[22]}$. Therefore, HIT antibody detection can be utilized as a method of differential diagnosis targeted at patients 
suspected of having HIT.Moreover, as the variations in HTI antibody concentration have a negative correlation with PLTc increase, this measure can be adopted as an index for predicting an improvement in the patient's response to clinical treatment. Although 5-hydroxytryptamine release from activated platelet function tests can be selected as a 'criterion' for HIT diagnosis, it is seldom applied because of methodological defects ${ }^{[23]}$.

Undoubtedly, there are certain limitations in the present study. As single-center observational research, laboratory tests were not conducted to determine HIT function.

\section{CONCLUSIONS}

Patients with HIT after surgery for acute type A aortic dissection have significantly increased postoperative complications and prolonged mechanical ventilation time and length of ICU stay, and the incidence of thromboembolism increased significantly. When there is an unexplained reduction in PLTc after surgery, it is necessary to be alert to the occurrence of HIT, especially when the time of cardiopulmonary bypass and aortic cross-clamping are long and the heparin dose is too large.HIT antibody testing and risk stratification should be performed for these early clinical high-risk patients.

This research was supported by the National Natural Science Foundation of China No.81970210,and the Fund of "Sanming" Project of Medicine in Shenzhen No.SZSM201612048.

\section{REFERENCES}

[1] Fathi M. Heparin-induced thrombocytopenia (HIT): Identification and treatment pathways.[J]. Global Cardiology Science and Practice, 2018, 2018(2).

[2] Girolami B, Prandoni P, Stefani P M, et al. The incidence of heparin-induced thrombocytopenia in hospitalized medical patients treated with subcutaneous unfractionated heparin: a prospective cohort study[J]. Blood, 2003, 101(8): 2955-2959.

[3] Salter B, Weiner M M, Trinh M, et al. Heparin-Induced Thrombocytopenia: A Comprehensive Clinical Review.[J]. Journal of the American College of Cardiology, 2016, 67(21): 2519-2532.

[4] Linkins LA, Dans AL, Moores LK, et a1. Treatment and prevention of heparin. Induced thrombocytopenia: Antithrombotic Therapy and Prevention of Thrombosis, 9th ed:American College of Chest Physicians Evidence-Based Clinical Practice Guidelines[J]. Chest, 2012, 141(2 Suppl): e495S-e530S. 
[5] Nagler M, Cuker A. Profile of Instrumentation Laboratory's HemosIL® AcuStar HIT-Ab(PF4-H) assay for diagnosis of heparin-induced thrombocytopenia[J]. Expert Review of Molecular Diagnostics, 2017, 17(5): 419-426.

[6] Tardy-Poncet B, Piot M, Montmartin A, et al. Delayed-onset heparin- induced thrombo-cytopenia without thrombosis in a patient receiving postoperative thromboprophylaxis with rivaroxaban[J]. Thromb Hae- most, 2015, 114(3): 652-654.

[7] Khandelwal S, Arepally G M. Immune pathogenesis of heparin-induced thrombocytopenia[J]. Thrombosis and Haemostasis, 2016, 116(5): 792-798.

[8] Karhausen J, Smeltz A M, Akushevich I, et al. Platelet Counts and Postoperative Stroke After Coronary Artery Bypass Grafting Surgery[J]. Anesthesia \& Analgesia, 2017, 125(4): 1129-1139.

[9] Cios T J, Salamancapadilla Y, Guvakov D V, et al. An Anti-Coagulation Conundrum: Implantation of Total Artificial Heart in a Patient with Heparin-Induced Thrombocytopenia Type II.[J]. American Journal of Case Reports, 2017: 294-298.

[10] Padmanabhan A, Jones C G, Bougie D W, et al. Heparin-independent, PF4-dependent binding of HIT antibodies to platelets: implications for HIT pathogenesis[J]. Blood, 2015, 125(1): 155-161.

[11] Lo GK, Juhl D, Warkentin TE et al: Evaluation of pretest clinical score (4 T's) for the diagnosis of heparin-induced thrombocytopenia in two clinical settings.[J]. Thromb. Haemost, 2006; 4: 759-765.

[12] Watson H G, Davidson S J, Keeling D, et al. Guidelines on the diagnosis and management of heparin-induced thrombocytopenia: second edition[J]. British Journal of Haematology, 2012, 159(5): 528-540.

[13] Cuker A, Gimotty P A, Crowther M A, et al. Predictive value of the 4Ts scoring system for heparin-induced thrombocytopenia: a systematic review and meta-analysis[J]. Blood, 2012, 120(20): 4160-4167.

[14] Parolari A, Tremoli E, Songia P, et al. Biological features of thoracic aortic diseases. Where are we now, where are we heading to: established and emerging biomarkers and molecular pathways[J]. European Journal of Cardio-Thoracic Surgery, 2013, 44(1): 9-23.

[15] Bossone E, Pyeritz RE, O'Gara P,et a1. Acute aortic dissection in blacks: insights from the International Registry of Acute Aortic Dissection.[J]. Am J Med, 2013, 126: 909-915.

[16] Azab B, Shah N, Akerman M, et al. Value of platelet/lymphocyte ratio as a predictor of all-cause mortality after non-ST-elevation myocardial infarction.[J]. Journal of Thrombosis and Thrombolysis, 2012, 34(3): 326-334.

[17] Wahba A, Black G, Koksch M, et al. Cardiopulmonary bypass leads to a preferential loss of activated platelets. A flow cytometric assay of platelet surface antigens.[J]. European Journal of Cardio-Thoracic Surgery, 1996, 10(9): 768-773. 
[18] Agarwal S, Johnson R I, Kirmani B H, et al. Pre- and Post-Bypass Platelet Function Testing With Multiple Electrode Aggregometry and TEG Platelet Mapping in Cardiac Surgery[J]. Journal of Cardiothoracic and Vascular Anesthesia, 2015, 29(5): 1272-1276.

[19] Linkins L, Bates S M, Lee A Y, et al. Combination of 4Ts score and PF4/H-PaGIA for diagnosis and management of heparin-induced thrombocytopenia: prospective cohort study[J]. Blood, 2015, 126(5): 597-603.

[20] Salter B, Weiner M M, Trinh M, et al. Heparin-Induced Thrombocytopenia: A Comprehensive Clinical Review.[J]. Journal of the American College of Cardiology, 2016, 67(21): 2519-2532.

[21] Legnani C, Cini M, Pili C, et al. Evaluation of a new automated panel of assays for the detection of anti-PF4/heparin antibodies in patients suspected of having heparin-induced thrombocytopenia[J]. Thrombosis and Haemostasis, 2010, 104(2): 402-409.

[22] Nagler M, Bachmann L M, Cate H T, et al. Diagnostic value of immunoassays for heparin-induced thrombocytopenia: a systematic review and meta-analysis[J]. Blood, 2016, 127(5): 546-557.

[23] Cuker A, Arepally G M, Chong B H, et al. American Society of Hematology 2018 guidelines for management of venous thromboembolism: heparin-induced thrombocytopenia[J]. Blood Advances, 2018, 2(22): 3360-3392. 
Figures

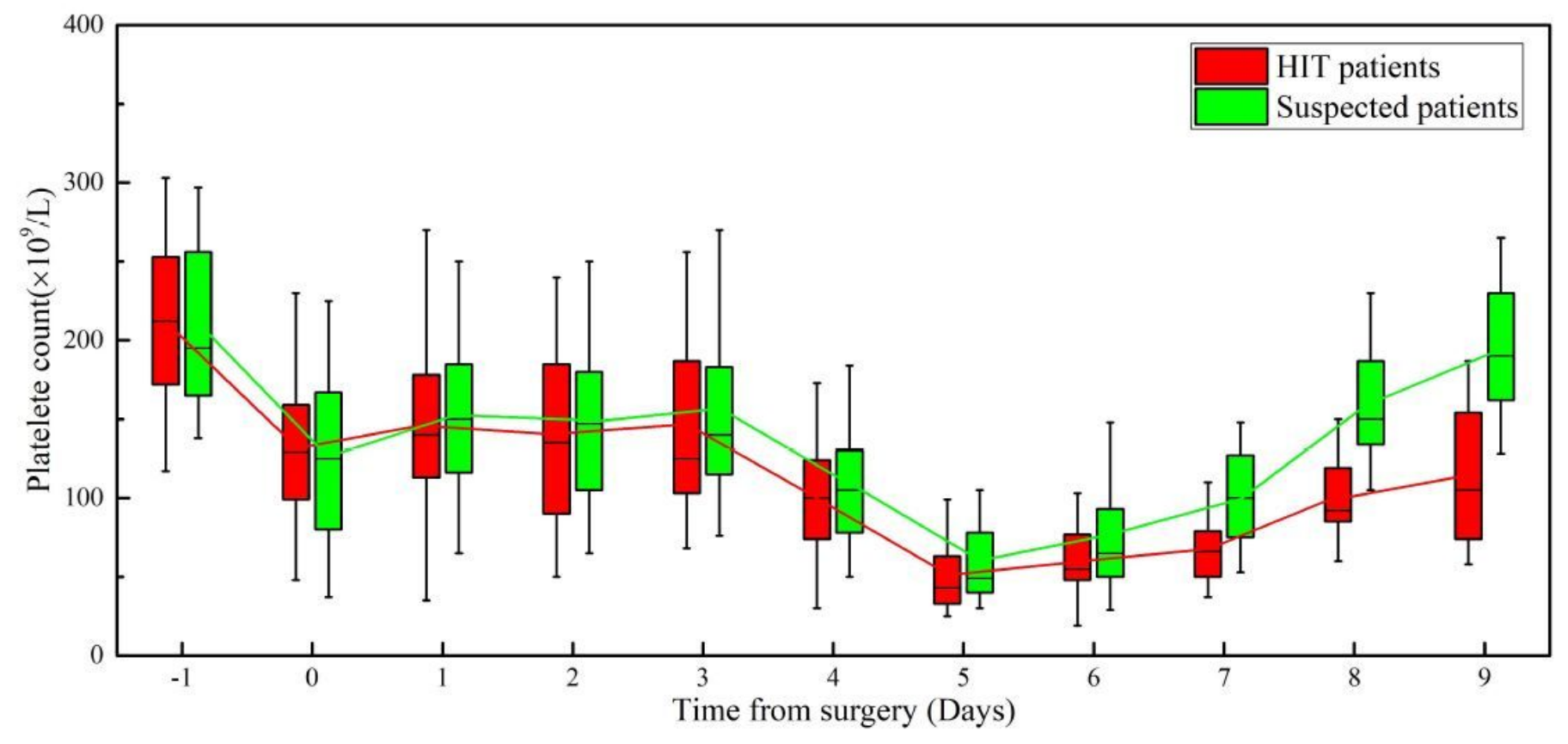

Figure 1

Comparison of platelet counts between HIT patients and Suspected HIT patients. 


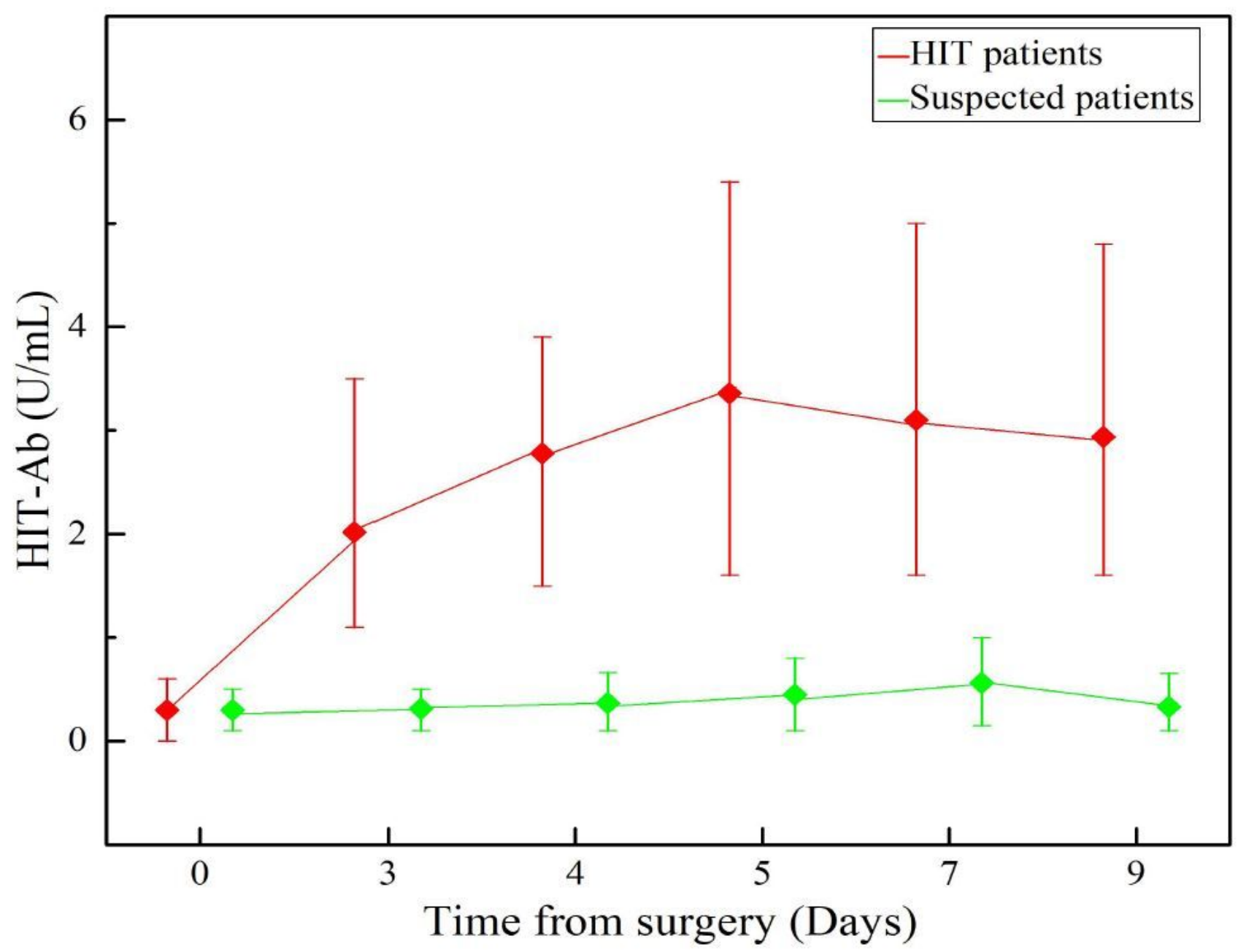

Figure 2

Comparison of HIT-Ab between HIT patients and Suspected HIT patients.

\section{Supplementary Files}

This is a list of supplementary files associated with this preprint. Click to download.

- Declarations.docx 